\title{
Robot Foragers
}

\section{Using a swarm of puck-shaped robots, researchers simulate interactions between biological organisms and their environment.}

\author{
By Michael Schirber
}

A swarm of small robots can imitate deer or bacteria searching for food. Researchers released these electronic foragers on an LED screen whose light output was adjusted to mimic the amount of a consumable resource-such as grass for deer [1]. Once the robots "ate" the resource in one place, they moved out of the depleted region. This programmed behavior led to collective patterns that resembled phases of matter including liquid, crystal, and glass. The team plans to further explore self-organization in the robots by giving them additional traits, such as a preference for consuming specific colors of light.

"Active matter" refers to systems consisting of many elements that move on their own. Biological examples include bacterial colonies and bird flocks, which researchers try to imitate with

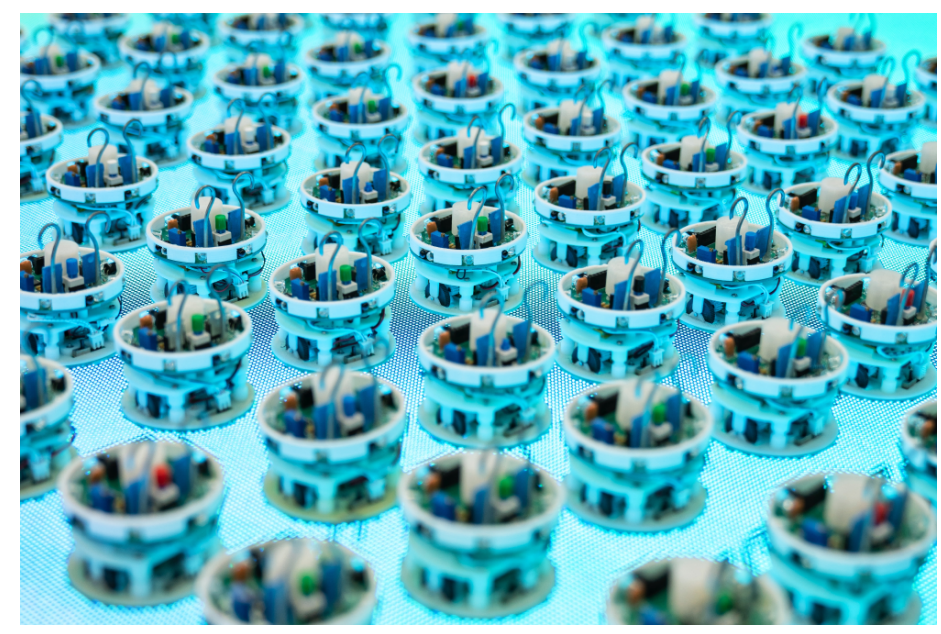

Robot herd. Disk-shaped robots are programmed to move toward light, mimicking the foraging behaviors of bacteria and other organisms. (See video below.)

Credit: L. Liu/Chongqing Univ. artificial active elements. Previous experiments have used robots or self-propelled particles that move in response to some physical input, such as light or chemical concentrations (see Focus: Sensing Delays Control Robot Swarming). But in a real ecological system, the environment is not some fixed lab table-it constantly changes in response to the activity of organisms. And the organisms must adapt to the changes they induce in their environment. Bacteria, for example, will consume nutrients and thus change the concentrations of chemicals in their surroundings. "They can sense that they are in a depleted region and will move in the direction of more food," says Robert Austin from Princeton University.

It is possible to design computer simulations that include hungry organisms living in a resource-limited environment. However, Austin believes that simulations can't capture the collective, or "emergent" behaviors that arise in biological active matter. "Biological objects can have a complexity that cannot be simulated by a digital computer," Austin says. He partnered with Liyu Liu of Chongqing University in China and other colleagues to design an analog experiment that lets biology-inspired robots explore and shape their environment.

The team fabricated 8-cm-wide hockey-puck-shaped robots that had wheels and light sensors on their underbellies. The robots were programmed to move in response to light from below, always heading toward brighter light, in the direction in which the brightness increased the fastest. The researchers placed 50 of these robots on a $4 \mathrm{~m} \times 4 \mathrm{~m}$ LED light board whose pixels could be individually controlled. The light underneath each robot was dimmed over a certain spatial region and for a chosen time duration, mimicking how a resource is consumed and later recovers. The robots responded to the dimming by rolling in the brightest direction, and then their new locations were dimmed. The team calls the robots' gradient-guided 


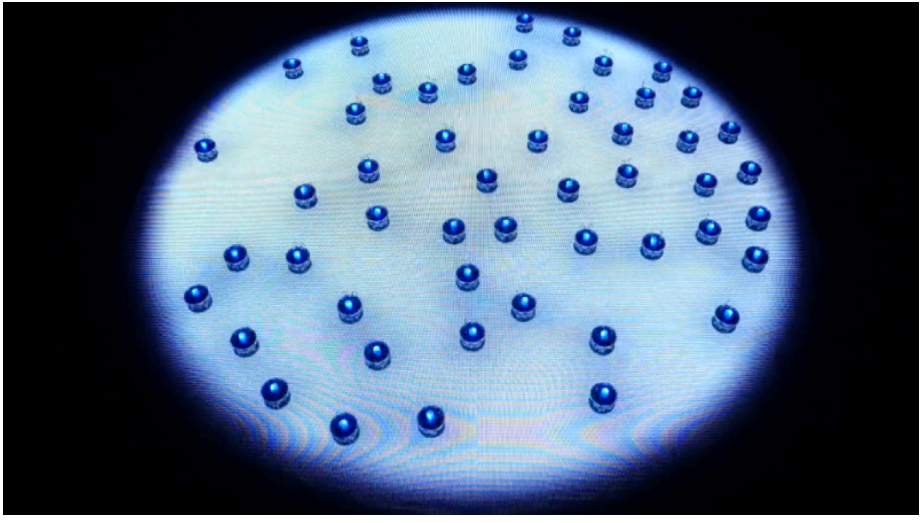

The collective behavior of "light-eating" robots is explored in a shrinking environment. In the beginning, the robots move around randomly, as in a gas. They eventually form a crystal-like hexagonal pattern, with each robot "dancing" with six partners in a ring. Toward the end, the shrunken "dance floor" dims-from the robot light-consumption-and the movement becomes liquid-like and then glass-like. The video is sped up by $68 \times$.

Credit: G. Wang et al. [1]

motion a "warp drive," as it is based on a warping of the "resource space" around a robot [2].

The team showed that the robots repel one another, as they are attracted to bot-free regions, where "food" is more plentiful. The researchers explored the effects of these interactions by creating a circular light environment, surrounded by darkness. At the outset, the circle was large, and the robots moved around randomly, like atoms in a gas. But as the researchers shrunk the environment by reducing the circle's diameter, the robots packed together into a hexagonal crystalline pattern, with each bot confined to a little patch surrounded by six neighbors. With further shrinking, the depleted regions began to overlap, forming a uniform light landscape where no region was more attractive than any other. This smoothing of the landscape caused the crystal to "melt" into a liquid-like phase, followed by a disordered, glass-like phase, in which the robots became locked in place as they contacted one another.

Austin says this emergent behavior has not been seen in previous active matter experiments. He hopes that upgrades of the robots could reveal biologically relevant behavior. To this end, his team has begun including color sensitivity, with some robots foraging for, say, red light, while others consume blue light. These color traits could then be passed from one robot to another in a robotic version of reproduction. The researchers imagine these gene-carrying robots could provide insight into the development of tumor cells, for example. "Robotic active matter is an uncultivated land, full of potential," Liu says.

"The novelty of this work lies in the clever and simple experimental realization, with control of the rates of both grazing and regrowth," says active-matter physicist Sriram Ramaswamy from the Indian Institute of Science in Bengaluru, India. He thinks the system offers many potential directions for exploration, such as studying how the environment may go through its own phase transitions in response to interactions with the community of organisms.

Michael Schirber is a Corresponding Editor for Physics based in Lyon, France.

\section{REFERENCES}

1. G. Wang et al., "Emergent field-driven robot swarm states," Phys. Rev. Lett. 126, 108002 (2021).

2. T. Phan et al., "Bootstrapped motion of an agent on an adaptive resource landscape," Symmetry 13, 225 (2021). 